(2) Open Access Full Text Article

\title{
Association of depression and anxiety before heart transplant with mortality after transplant: a single-center experience
}

This article was published in the following Dove Press journal:

Transplant Research and Risk Management

24 March 2017

Number of times this article has been viewed

\author{
Flavio Epstein' \\ Melissa M Parker ${ }^{2}$ \\ Anna Lucero 3 \\ Rakesh Chaudhary ${ }^{4}$ \\ Eyun Song ${ }^{3}$ \\ Dana Weisshaar'
}

'Heart Transplant Department, Kaiser Permanente Santa Clara Medical Center, Santa Clara, ${ }^{2}$ Kaiser Permanente Division of Research, Oakland, ${ }^{3}$ Department of Graduate Medical Education, ${ }^{4}$ Department of Internal Medicine, Kaiser Permanente Santa Clara Medical Center, Santa Clara, CA, USA
Correspondence: Flavio Epstein Heart Transplant Department, Kaiser Permanente Santa Clara Medical Center, Homestead Campus, 3rd Floor, Department 342, 710 Lawrence Expressway, Santa Clara, CA 9505I, USA Tel +l 4088513329

Email flavio.x.epstein@kp.org
Objective: The purpose of this study was to evaluate the effects of depression and anxiety before heart transplant on all-cause mortality after heart transplant in a Northern California cohort.

Methods: A total of 130 adult patients with heart transplants enrolled at Kaiser Permanente between June 2005 and December 2013 were included in a retrospective chart review. Preoperative depression and anxiety, evidenced by diagnoses, and other risk factors for all-cause mortality were investigated. Statistical methods included Kaplan-Meier survival analysis and Cox proportional hazard regression models.

Results: After risk adjustment, patients with preoperative depression and anxiety diagnoses had higher risk of all-cause mortality at 2 years (hazard ratio $[\mathrm{HR}]=4.2,95 \%$ confidence interval $[\mathrm{CI}]$ : $1.1,15.0, p=0.03)$ and 3 years $(\mathrm{HR}=3.7,95 \% \mathrm{CI}: 1.2,11.9, p=0.04)$ following heart transplant than those without depression or anxiety. This finding did not reach statistical significance at 5 years post-heart transplant ( $\mathrm{HR}=2.0,95 \% \mathrm{CI}: 0.8,5.3, p=0.14)$.

Conclusion: The findings suggest an association between preoperative depression and anxiety with mortality in heart transplant patients 2 and 3 years post-transplant.

Keywords: pre-operative depression, anxiety, survival after heart transplant

\section{Introduction}

Approximately 2,000 heart transplants were performed in adult patients in the United States each year between 2005 and 2007. Average survival was $88 \%$ at 1 year, $81 \%$ at 3 years, and $75 \%$ at 5 years. ${ }^{1}$ The leading causes of death in post-heart transplant patients were cardiac allograft vasculopathy (CAV), malignancy (non-lymphoma/posttransplant lymphoproliferative disorder), graft failure, renal failure, acute rejection, infection (non-cytomegalovirus), and multiple organ failure. ${ }^{2}$

This study was designed to evaluate the effects of depression and anxiety before heart transplant on mortality after transplant and to identify risk factors for mortality post-transplant. Depression has been studied as a chronic emotional state that increases cardiac risk, and it has been associated with morbidity and mortality in heart failure patients..$^{3-8}$ The need for a psychological evaluation pre-transplantation has been endorsed in previous studies. ${ }^{9-13}$ The evaluation may assist in mental health diagnosis and treatment, improving quality of life before and after heart transplantation. Up to $60 \%$ of patients in the transplant evaluation period meet the criteria for a depression and/or anxiety diagnosis. ${ }^{10}$

A recent meta-analysis of 20 studies concluded that "depression increases risk for post-transplant mortality" and calculated a pooled relative risk (RR) of 1.65 
(95\% confidence interval $[\mathrm{CI}]: 1.3,2.0)$; however, no significant association was found between anxiety and mortality (pooled $\mathrm{RR}=1.39,95 \% \mathrm{CI}: 0.9,2.3$ ) in six small studies. ${ }^{14}$ Since organ transplants are uncommon, studies in this patient population generally have small sample sizes, which poses a challenge in terms of generalizability and reproducibility of results.

Our study adds to the existing literature by evaluating the association of pre-transplant depression and anxiety, alone and jointly, with all-cause mortality following heart transplant in an integrated managed health care setting.

\section{Methods}

In this retrospective cohort study, we evaluated the association between pre-transplant depression and anxiety and post-transplant mortality in heart transplant recipients and identified risk factors associated with death after transplant. Study subjects were members of Kaiser Permanente Northern California (KPNC) health plan who received a heart transplant between June 2005 and December 2013. KPNC is a large, integrated health care delivery system that provides care to $\sim 3.9$ million patients.

Eligible study subjects were KPNC members for at least 6 months prior to the date of transplant and at least 17 years old on the date of the transplant. A total of 130 patients met the study criteria. The majority of patients were first-time recipients. Two (1.5\%) patients underwent re-transplantation, and six (4.6\%) received dual organ (heart-kidney) transplantation. Patients were followed up for a maximum of 5 years post-transplant, until death, disenrollment from the health plan, or through December 31, 2014, whichever came first.

Data were collected via review of the electronic medical records (EMRs) and extracted from administrative and clinical databases maintained by the health plan. The main exposure variables were depression and anxiety status in the year before the heart transplant.

Depression and anxiety were identified by at least one outpatient mental health diagnosis in the outpatient setting by any physician or other health care providers, including nurse practitioners and licensed mental health providers, in the year prior to transplantation. Referrals to primary care and psychiatry were routinely made for treatment, and their care was integrated with the heart transplant service providers' care.

In addition to the diagnoses, we also recorded 1) any psychotropic medication and the prescription date(s) and 2) patient self-report instruments (Beck Depression Inventory-II [BDI-II] and State and Trait Anxiety Inventory [STAI]) recorded in the EMR in the year prior to transplant.
On the BDI-II, a score of $\geq 14$ based on Beck's scoring system supported a diagnosis of depression. ${ }^{15}$ On the STAI, a score of $\geq 49$ on the State Anxiety and $\geq 44$ on the Trait Anxiety supported a diagnosis of anxiety. ${ }^{16}$ The BDI-II and the STAI scores were routinely obtained by the transplant psychologist during the pre-heart transplant evaluation period, and $77 \%$ ( 80 patients) had completed both the BDI-II and the STAI.

The medication prescription history and the self-report instruments were used to gauge the severity and length of the depression and anxiety symptoms in the year pre-heart transplant. All patients with a depression and/or anxiety diagnoses, which mainly included adjustment disorders, major depressive disorder, generalized anxiety disorder, and depressive and anxiety disorders not otherwise specified (Diagnostic and Statistical Manual of Mental Disorders IV [DSM-IV]), had a valid prescription for either an anti-depressant or a benzodiazepine medication at transplant. Only 16 patients did not have a valid psychotropic medication prescription in the EMR at transplant. In addition to the data outlined earlier, we reviewed chart notes and noted that most patients experienced depression and/or anxiety for several months while they were getting psychotropic medication treatment in the year before heart transplant.

A clinical psychologist conducted the chart review and categorized each patient based on the protocol described earlier. Subjects were categorized as having no depression or anxiety, depression alone, anxiety alone, or both depression and anxiety.

We collected demographic data (age, gender, and race/ ethnicity), baseline clinical and comorbid conditions, including body mass index (BMI), renal dysfunction, diabetes, hypertension, ischemic heart disease, number of hospitalizations in the 6 months prior to the transplant, smoking status, and frequency of alcohol use. Data collected that were specific to the transplant included transplant waiting time, donor age, heart transplant listing status, and length of transplant hospitalization.

In the post-transplant period, we identified the following: cardiac allograft vasculopathy (CAV), the number of moderate or severe rejection episodes (Grade 2R or greater), need for re-transplant, and mortality. The main outcome variable for the predictive model was a combined measure of re-transplant and death. We opted to combine these two measures into one outcome for the predictive model since both are equally important adverse outcomes and re-transplant was too infrequent $(n=2)$ in this sample to study on its own.

We described the demographic and clinical characteristics of the cohort overall and stratified by depression/anxiety 
status using frequency counts and percentages for categorical variables and mean (standard deviation) for continuous variables. We used the Kaplan-Meier method to produce survival curves measuring time to death or re-transplant. The log-rank chi-square test was used to test for differences in survival between each of the three exposure categories (both depression and anxiety, depression alone, and anxiety alone) and the reference group (no depression or anxiety). $p$-Values were adjusted for multiple comparisons using the Bonferroni adjustment method. Finally, Cox proportional hazard regression models were used to estimate the effects of depression/anxiety status on the combined outcome of either mortality or re-transplant.

We ran unadjusted as well as multivariate models that adjusted for known potential confounders: age ( $\geq 55$ versus $<55$ years old), gender, race/ethnicity, renal dysfunction, diabetes, hypertension, and heart transplant listing status. We conducted a sensitivity analysis that dichotomized the exposure into two categories: any depression or anxiety versus none. We also reported on the effect of these covariates as independent risk factors for post-transplant mortality. The proportionality assumption was tested and verified with Shoenfeld residuals. We ran models examining the outcomes at 2, 3, and 5 years post-heart transplant.

We were unable to conduct a similar analysis at 1 year post-transplant because none of the patients in our reference population died or had a re-transplant in the first year. Patients without depression and anxiety had the best survival in the first year, compared to the other groups. We considered a $p$-Value of $<0.05$ to be statistically significant.

This study was approved by the Kaiser Foundation Research Institute's Institutional Review Board (KFRI IRB), and the study was approved with a waiver of consent due to its retrospective design.

\section{Results}

Of the 130 heart transplant patients included in the study, 50 (38.5\%) had no evidence of depression or anxiety within the 1 year prior to transplant, $22(17 \%)$ had depression only, 13 $(10 \%)$ had anxiety only, and $45(34.5 \%)$ had both depression and anxiety (Table 1). The mean age in the cohort was 52 years. Seventy percent $(n=90)$ of the patients were male, and almost half (48\%) were minorities, indicating a diverse cohort of patients. Asian/Pacific Islander was the most common racial minority $(n=21)$, followed by Black $(n=16)$ and Hispanic $(n=14)$. There were no statistically significant differences in demographic characteristics and baseline clinical or comorbid conditions across depression and anxiety categories.
The depression with anxiety group's means for the BDI-II and STAI instruments were higher than those reported by the Florida Cardiac Cohort Normative Descriptive Measures (14): BDI-II $=13.9$ (versus 10.58), State Anxiety $=40.2$ (versus 37.26), and Trait Anxiety =40.6 (versus 34.57), suggesting worse depression and anxiety severity in our patients with both depression and anxiety compared to a broad heart failure group.

The BDI-II and STAI scores were consistent with the presence of a depression and/or anxiety diagnosis, the prescription of psychotropic medication, and the clinical notes in the EMR. Of the 80 patients with a depression and/or anxiety diagnosis within 1 year prior to transplant, 64 (80\%) had a valid prescription for either an anti-depressant or a benzodiazepine medication at the time of transplant.

Pre-transplant prevalence of diabetes was $29.2 \%$, hypertension $43.9 \%$, renal dysfunction $48.8 \%$, and ischemic heart disease $31.5 \%$. The mean number of hospitalizations in the 6 months prior to heart transplant was 2.5 , mean body mass index was close to normal $\left(26.5 \mathrm{~kg} / \mathrm{m}^{2}\right)$, and the prevalence rates of history of smoking and alcohol use were $13.1 \%$ and $9.2 \%$, respectively.

Nine patients $(6.9 \%)$ died during the year following heart transplant surgery (Figure 1, Table 2). Overall, survival at 1 year post-heart transplant was $93.1 \%$. Patients with depression only, anxiety only, and both depression and anxiety experienced higher mortality than patients without depression and anxiety, $9.1 \%, 7.7 \%, 13.3 \%$, and $0 \%$, respectively. In the univariate analysis, there was a statistically significant difference in 1-year survival between the groups with both depression and anxiety compared to those with neither.

Patients with both depression and anxiety in the year prior to cardiac transplantation experienced significantly higher rates of post-transplant mortality compared to patients without either of these diagnoses $(p=0.01)$. The cumulative probability of mortality at 2, 3, and 5 years post-heart transplant was $12.6 \%, 14.8 \%$, and $22.8 \%$; however, these differences were not statistically significant (Table 3). Malignancy (19\%), infection (15\%), and nonspecific graft failure over 30 days $(15 \%)$ were the top three leading causes of death in this heart transplant cohort (Table 4).

Clinical confounders were identified based on existing literature and the International Society for Heart and Lung Transplantation (ISHLT) registries (age, gender, race/ethnicity, renal dysfunction, diabetes, hypertension, and heart transplant listing status). After adjustment for these clinical confounders and demographics, depression with anxiety (higher distress) was strongly associated with mortality or 
Table I Patient characteristics

\begin{tabular}{|c|c|c|c|c|c|}
\hline Characteristics & $\begin{array}{l}\text { No Dep } \\
\text { and Anx } \\
(n=50)\end{array}$ & $\begin{array}{l}\text { Dep only } \\
(n=22)\end{array}$ & $\begin{array}{l}\text { Anx only } \\
(n=13)\end{array}$ & $\begin{array}{l}\text { Dep } \\
\text { and Anx } \\
(n=45)\end{array}$ & $\begin{array}{l}\text { p-Value* } \\
\text { (no Dep and Anx } \\
\text { versus Dep and Anx) }\end{array}$ \\
\hline \multicolumn{6}{|l|}{ Demographics } \\
\hline Age $($ mean $\pm S D)$ & $51 \pm 16.3$ & $56 \pm 11.7$ & $48 \pm 16.9$ & $52 \pm 12.6$ & 0.64 \\
\hline \multicolumn{6}{|l|}{ Gender, n (\%) } \\
\hline Male & $4 I(82)$ & $14(64)$ & $4(3 I)$ & $32(7 I)$ & \multirow{2}{*}{0.21} \\
\hline Female & $9(18)$ & $8(36)$ & $9(69)$ & $13(29)$ & \\
\hline \multicolumn{6}{|l|}{ Race/ethnicity, n (\%) } \\
\hline White & $22(44)$ & $13(59)$ & $10(77)$ & $23(5 \mathrm{I})$ & \multirow{5}{*}{0.8} \\
\hline Black & $8(16)$ & $2(9)$ & I (8) & $5(I I)$ & \\
\hline Hispanic & $5(10)$ & $3(14)$ & $0(0)$ & $6(13)$ & \\
\hline Asian/Pacific Islander & $8(16)$ & $4(18)$ & $2(15)$ & $7(16)$ & \\
\hline Mixed & $7(17)$ & $0(0)$ & $0(0)$ & $4(9)$ & \\
\hline \multicolumn{6}{|l|}{ Pre-transplant risk factors } \\
\hline BDI score (mean $\pm S D)$ & $6.9 \pm 2.4$ & $12.6 \pm 6.9$ & $7.4 \pm 4.2$ & $13.9 \pm 6.7$ & $<0.0001$ \\
\hline State score $($ mean $\pm S D)$ & $28.4 \pm 6.6$ & $29.4 \pm 8.3$ & $33.6 \pm 10.1$ & $40.2 \pm 12.1$ & $<0.0001$ \\
\hline Trait score (mean $\pm S D)$ & $29.0 \pm 6.2$ & $27.2 \pm 5.6$ & $32.2 \pm 7.3$ & $40.6 \pm 11.5$ & $<0.0001$ \\
\hline BMI (mean \pm SD) & $26.2 \pm 4.7$ & $26.5 \pm 4.7$ & $25.1 \pm 4.0$ & $27.2 \pm 5.1$ & 0.33 \\
\hline Renal dysf, n (\%) & $25(50)$ & $14(64)$ & $4(31)$ & $20(44)$ & 0.52 \\
\hline Number of hospitalizations 6 months post-HT, n (\%) & $2.7(2.0)$ & $2.3(1.7)$ & $2.2(1.9)$ & $2.4(1.4)$ & 0.37 \\
\hline Smoking, n (\%) & $8(16)$ & $2(9)$ & $0(0)$ & $7(16)$ & 0.95 \\
\hline Alcohol use, n (\%) & $3(6)$ & $2(9)$ & $0(0)$ & $7(16)$ & 0.13 \\
\hline Diabetes, n (\%) & $14(28)$ & $7(32)$ & $2(15)$ & $15(33)$ & 0.57 \\
\hline Hypertension, n (\%) & $23(46)$ & $10(45)$ & $4(31)$ & $20(44)$ & 0.88 \\
\hline Ischemic, n (\%) & $19(38)$ & $8(36)$ & $2(15)$ & $12(27)$ & 0.24 \\
\hline Donor age (mean $\pm \mathrm{SD})$ & $3 I \pm I I .5$ & $35 \pm 13.7$ & $28 \pm 10.3$ & $3 I \pm I I .2$ & 0.89 \\
\hline Wait time in days (mean \pm SD) & $113 \pm 342$ & $68 \pm 78$ & $8 I \pm 50$ & $113 \pm 137$ & 0.99 \\
\hline \multicolumn{6}{|l|}{ UNOS heart transfer status, $\mathrm{n}(\%)$} \\
\hline IA & $14(28)$ & $7(32)$ & $2(15)$ & $13(29)$ & \multirow{4}{*}{0.98} \\
\hline IB & $28(56)$ & $8(36)$ & $9(69)$ & $27(60)$ & \\
\hline 2 & $6(12)$ & $7(32)$ & $2(15)$ & $5(\mathrm{II})$ & \\
\hline Unknown & $2(4)$ & $0(0)$ & $0(0)$ & $0(0)$ & \\
\hline \multicolumn{6}{|l|}{ Post-transplant risk factors } \\
\hline \multicolumn{6}{|l|}{ Rejection episode } \\
\hline Moderate, n (\%) & I (2) & I (5) & $0(0)$ & $2(4)$ & 0.9 \\
\hline Severe, n (\%) & $20(40)$ & II (50) & $8(62)$ & $22(49)$ & 0.37 \\
\hline Re-transplant, n (\%) & I (2) & $0(0)$ & $I(8)$ & $0(0)$ & 0.53 \\
\hline CAV, n (\%) & $18(36)$ & $10(45)$ & $2(15)$ & $12(27)$ & 0.33 \\
\hline LOS in days (mean \pm SD) & $14 \pm 8.1$ & $20 \pm 18.4$ & $\mathrm{II} \pm 5.7$ & $13 \pm 7.3$ & 0.55 \\
\hline
\end{tabular}

Notes: *Chi-square (or Fisher's exact test when cell size $<5$ ) for categorical and $t$-test for continuous variables (two-tailed test).

Abbreviations: Dep, depression; Anx, anxiety; SD, standard deviation; BDI, Beck Depression Inventory; BMI, body mass index; dysf, dysfunction; HT, heart transplant; UNOS, United Network for Organ Sharing; CAV, cardiac allograft vasculopathy; LOS, length of stay.

re-transplant at 2 years (hazard ratio $[\mathrm{HR}]=4.2,95 \% \mathrm{CI}$ : $1.1,15.0, p=0.03)$ and 3 years $(\mathrm{HR}=3.7,95 \% \mathrm{CI}: 1.2,11.9$, $p=0.04)$ but not at 5 years post-heart transplant $(\mathrm{HR}=2.0$, 95\% CI: 0.8, 5.3, $p=0.14$ ) (Table 3). The multivariate analysis was not performed for the 1-year time frame since no deaths occurred in the reference group. Individually, depression alone and anxiety alone did not have statistically significant association with mortality. However, when we modeled the exposure as any depression or anxiety (i.e., depression alone, anxiety alone, or both depression and anxiety), we also found a strong association with mortality and re-transplant at 2 years $(\mathrm{HR}=4.2,95 \% \mathrm{CI}: 1.2,14.7, p=0.02)$ and 3 years $(\mathrm{HR}=3.6,95 \% \mathrm{CI}: 1.2,11.1, p=0.03)$ but not at 5 years postheart transplant.

Additional independent risk factors for mortality and re-transplant at 2 and 3 years post-heart transplant were male gender, Black and Asian race/ethnicity, and renal dysfunction (Table 3). Interestingly, pre-transplant hypertension was associated with decreased risk of mortality and re-transplant at 2,3 , and 5 years post-heart transplant $(\mathrm{HR}=0.2, p \leq 0.03)$. 
5-Year overall survival in all patients by pre-heart transplant depression and anxiety

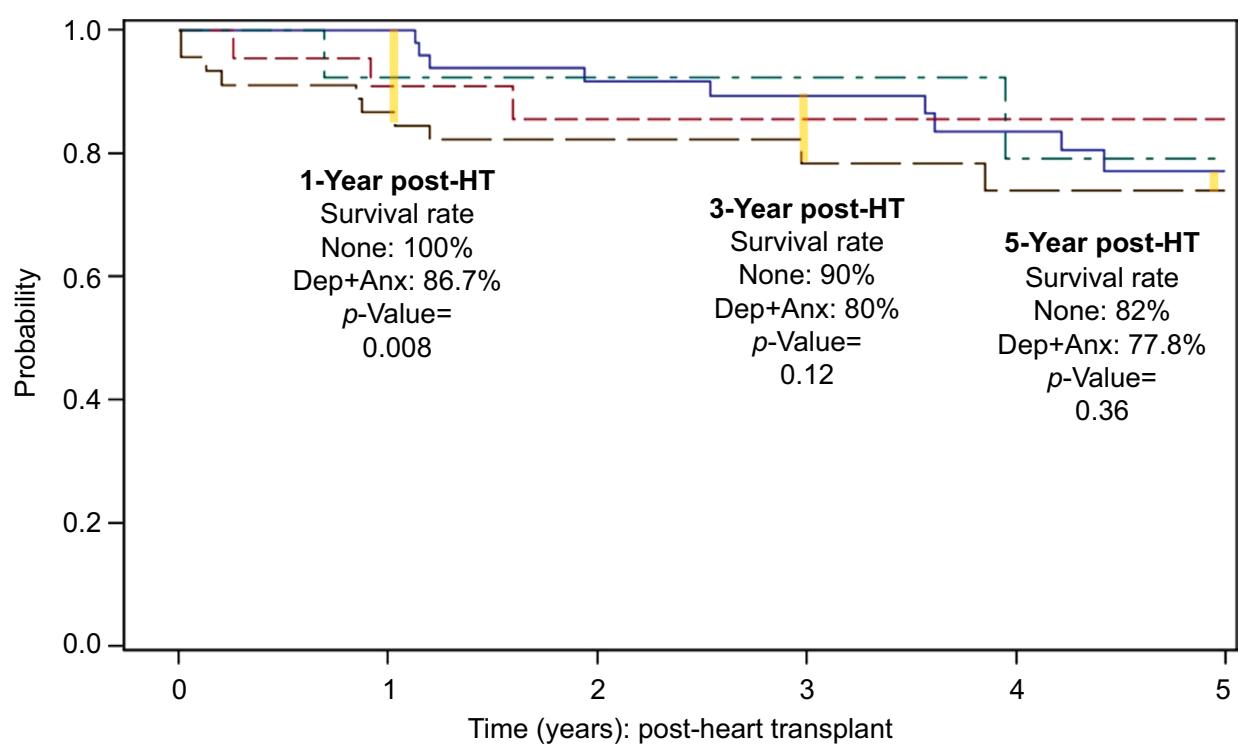

\begin{tabular}{l}
- Cohort \\
- (0) No depression or anxiety $\quad---$ (1) Depression only \\
-- (2) Anxiety only \\
\hline
\end{tabular}

Figure I Kaplan-Meier survival analysis: overall survival (years).

Abbreviations: HT, heart transplant; Dep, depression; Anx, anxiety.

Table 2 Kaplan-Meier survival analysis: I-year survival post-heart transplant

\begin{tabular}{|c|c|c|c|c|c|}
\hline Dep, Anx & Total, $\mathbf{n}$ & Events (\%) & Censored (\%) & Log-rank $p$-Value & $\begin{array}{l}\text { Cumulative } \\
\text { probability } \\
\text { of events (\%) }\end{array}$ \\
\hline \multicolumn{6}{|c|}{ I-Year post-heart transplant } \\
\hline No Dep and Anx & 50 & $0(0)$ & $50(100)$ & Ref & 0 \\
\hline Dep only & 22 & $2(9.1)$ & $20(90.9)$ & 0.06 & 9.1 \\
\hline Anx only & 13 & I (7.7) & $12(92.3)$ & 0.06 & 7.7 \\
\hline Dep and Anx & 45 & $6(13.3)$ & $39(86.7)$ & 0.01 & 13.3 \\
\hline All & 130 & $9(6.9)$ & $121(93.1)$ & - & 6.9 \\
\hline \multicolumn{6}{|c|}{ 2-Year post-heart transplant } \\
\hline No Dep and Anx & 40 & $4(8.0)$ & $46(92.0)$ & Ref & 8.4 \\
\hline Dep only & 22 & $3(13.6)$ & $19(86.4)$ & 0.33 & 14.4 \\
\hline Anx only & 12 & I (7.7) & $12(92.3)$ & 0.47 & 7.7 \\
\hline Dep and Anx & 45 & $8(17.8)$ & $37(82.2)$ & 0.12 & 17.8 \\
\hline All & 130 & $16(12.3)$ & $114(87.7)$ & - & 12.6 \\
\hline \multicolumn{6}{|c|}{ 3-Year post-heart transplant } \\
\hline No Dep and Anx & 50 & $5(10.0)$ & $45(90.0)$ & Ref & 10.7 \\
\hline Dep only & 22 & $3(13.6)$ & $19(86.4)$ & 0.42 & 14.4 \\
\hline Anx only & 13 & I (7.7) & $12(92.3)$ & 0.56 & 7.7 \\
\hline Dep and Anx & 45 & $9(20.0)$ & $36(80.0)$ & 0.12 & 21.7 \\
\hline All & 130 & $18(13.9)$ & $112(86.2)$ & - & 14.8 \\
\hline \multicolumn{6}{|c|}{ 5-Year post-heart transplant } \\
\hline No Dep and Anx & 50 & $9(18.0)$ & $41(82.0)$ & Ref & 22.9 \\
\hline Dep only & 22 & $3(13.6)$ & $19(86.4)$ & 0.96 & 14.4 \\
\hline Anx only & 13 & $2(15.4)$ & II (84.6) & 0.83 & 20.9 \\
\hline Dep and Anx & 45 & $10(22.2)$ & $35(77.8)$ & 0.37 & 26 \\
\hline All & 130 & $24(18.5)$ & $106(81.5)$ & - & 22.8 \\
\hline
\end{tabular}

Abbreviations: Dep, depression; Anx, anxiety; Ref, reference. 
Table 3 Predictors of mortality (Cox proportional hazard regression analysis-adjusted models)

\begin{tabular}{|c|c|c|c|c|c|c|}
\hline \multirow[t]{2}{*}{ Variables } & \multicolumn{2}{|l|}{ 2-Year mortality } & \multicolumn{2}{|l|}{ 3-Year mortality } & \multicolumn{2}{|l|}{ 5-Year mortality } \\
\hline & HR (95\% Cl) & $\overline{p \text {-Value }}$ & HR (95\% CI) & $p$-Value & HR (95\% Cl) & $p$-Value \\
\hline \multicolumn{7}{|l|}{ Psychological state } \\
\hline Any depression or anxiety & $4.23(1.22,14.67)$ & 0.02 & $3.59(1.16,11.12)$ & 0.03 & $1.89(0.77,4.66)$ & 0.16 \\
\hline No Dep and Anx & Ref & - & Ref & - & Ref & - \\
\hline Dep and Anx & $4.15(1.14,15.03)$ & 0.03 & $3.71(1.15,11.94)$ & 0.04 & $2.04(0.79,5.27)$ & 0.14 \\
\hline Anx Only & $5.53(0.47,65.36)$ & 0.17 & $4.01(0.37,43.61)$ & 0.25 & $2.27(0.39,13.13)$ & 0.36 \\
\hline Dep Only & $4.35(0.77,24.77)$ & 0.1 & $3.02(0.57,16.12)$ & 0.2 & $1.34(0.3 \mathrm{I}, 5.8 \mathrm{I})$ & 0.7 \\
\hline No Dep and Anx & Ref & - & Ref & - & Ref & - \\
\hline \multicolumn{7}{|l|}{ Demographics } \\
\hline Age $>55$ years & $0.72(0.23,2.25)$ & 0.57 & $0.84(0.29,2.45)$ & 0.76 & $1.14(0.46,2.83)$ & 0.78 \\
\hline Age $\leq 55$ years & Ref & - & Ref & - & Ref & - \\
\hline Male & $8.18(0.97,68.93)$ & 0.053 & $8.57(1.04,70.29)$ & 0.045 & $3.03(0.81,11.40)$ & 0.1 \\
\hline Female & Ref & - & Ref & - & Ref & - \\
\hline Black & $6.53(1.23,34.77)$ & 0.03 & 5.48 (I.II, 27.19) & 0.04 & $3.00(0.70,12.86)$ & 0.14 \\
\hline Hispanic & $2.21(0.40,12.21)$ & 0.37 & $2.46(0.57,10.68)$ & 0.23 & $2.00(0.56,7.21)$ & 0.29 \\
\hline Asian & $5.53(1.14,26.94)$ & 0.03 & $3.76(0.82,17.19)$ & 0.09 & $2.07(0.53,8.07)$ & 0.3 \\
\hline Mixed & $5.28(0.78,35.97)$ & 0.09 & $3.55(0.57,22.19)$ & 0.18 & $3.62(0.82,15.92)$ & 0.09 \\
\hline White & Ref & - & Ref & - & Ref & - \\
\hline \multicolumn{7}{|l|}{ Clinical characteristics } \\
\hline Renal dysfunction - yes & $3.97(1.16,13.57)$ & 0.03 & $3.24(1.08,9.78)$ & 0.04 & $2.43(0.98,6.02)$ & 0.06 \\
\hline Renal dysfunction - no & Ref & - & Ref & - & Ref & - \\
\hline Heart status IA & $0.60(0.19,1.97)$ & 0.4 & $0.77(0.25,2.33)$ & 0.67 & $0.6 \mathrm{I}(0.21,1.79)$ & 0.37 \\
\hline Heart status IB & Ref & - & Ref & - & Ref & - \\
\hline Diabetes - yes & $2.26(0.63,8.12)$ & 0.21 & $2.74(0.83,9.09)$ & 0.1 & $2.25(0.77,6.62)$ & 0.14 \\
\hline Diabetes - no & Ref & - & Ref & - & Ref & - \\
\hline Hypertension - yes & $0.24(0.07,0.89)$ & 0.03 & $0.19(0.06,0.67)$ & 0.01 & $0.18(0.06,0.56)$ & 0.003 \\
\hline Hypertension - no & Ref & - & Ref & - & Ref & - \\
\hline
\end{tabular}

Abbreviations: $\mathrm{HR}$, hazard ratio; $\mathrm{Cl}$, confidence interval; Dep, depression; Anx, anxiety; Ref, reference.

Table 4 COD (post-HT all)

\begin{tabular}{|c|c|c|c|c|c|}
\hline COD, n (\%) & $\begin{array}{l}\text { No Dep and } \\
\text { Anx }(n=10)\end{array}$ & $\begin{array}{l}\text { Dep only } \\
(n=4)\end{array}$ & $\begin{array}{l}\text { Anx only } \\
(n=2)\end{array}$ & $\begin{array}{l}\text { Dep and Anx } \\
(n=10)\end{array}$ & $\begin{array}{l}\text { Total } \\
(n=26)\end{array}$ \\
\hline Malignancy & $3(30)$ & $2(50)$ & $0(0)$ & $0(0)$ & $5(19)$ \\
\hline Infection & $2(20)$ & $0(0)$ & $0(0)$ & $2(20)$ & $4(15)$ \\
\hline Nonspecific graft failure $>30$ days post-HT & $I(I 0)$ & I (25) & I (50) & I (I0) & $4(15)$ \\
\hline Death of graft & $I(10)$ & $0(0)$ & $\mathrm{I}(50)$ & $0(0)$ & $2(8)$ \\
\hline Early graft failure within 30 days of transplant & $0(0)$ & $0(0)$ & $0(0)$ & $2(20)$ & $2(8)$ \\
\hline Sudden cardiac death & $0(0)$ & $0(0)$ & $0(0)$ & $2(20)$ & $2(8)$ \\
\hline Graft vasculopathy & $\mathrm{I}(10)$ & $0(0)$ & $0(0)$ & $0(0)$ & I (4) \\
\hline Multi-organ failure & $0(0)$ & $0(0)$ & $0(0)$ & I (I0) & I (4) \\
\hline Pulmonary embolism & $I(10)$ & $0(0)$ & $0(0)$ & $0(0)$ & I (4) \\
\hline Rejection - acute & $0(0)$ & $0(0)$ & $0(0)$ & $I(10)$ & I (4) \\
\hline Other & I (I0) & I (25) & $0(0)$ & I (10) & 3 (II) \\
\hline
\end{tabular}

Abbreviations: COD, cause of death; HT, heart transplant; Dep, depression; Anx, anxiety.

\section{Discussion}

Patients with life-threatening medical conditions are exposed to psychological stressors. Anxiety and depression are common among patients with end-stage heart disease who are being evaluated for heart transplant. In this retrospective cohort study, $>60 \%$ of transplant recipients had depression, anxiety, or both in the year prior to transplant. This is consistent with the findings of Engle. ${ }^{10}$
We found an association between pre-transplant depression with anxiety and post-transplant mortality up to 3 years post-transplant but not at 5 years post-transplant. We also found similar effect sizes between depression alone and anxiety alone on mortality at 2 or 3 years post-transplant, which did not reach statistical significance.

In our cohort, anxiety with depression was shown to be associated with mortality post-transplant, and a recent 
meta-analysis failed to report this association. ${ }^{14}$ Accurate documentation of anxiety diagnoses and treatment history may have contributed to this finding, whereas in previous studies, the effects of anxiety may have been washed out by the lack of documentation or a diagnosis separate from depression. However, this needs to be tested.

In contrast, in our cohort, depression with anxiety pretransplant was not statistically associated with mortality at 5 years. That may be due to higher risk for post-transplant mortality in the immediate 2- to 3-year period, which diminishes over time. Another explanation of the lack of association between depression with anxiety at 5 years might be that patients receive good medical care with better survival post-transplant, and therefore, depression and anxiety could be a more important risk factor in mortality than in a setting where all transplant patients are dying earlier because of other causes. These hypotheses need to be tested.

Finally, another hypothesis to explain the lack of statistical significance on the effect of depression with anxiety and mortality at 5 years might be the time frame itself. The median duration of follow-up of all the papers cited in the meta-analysis was 5.8 years. ${ }^{14}$ We did not see a relationship at 5 years. The risk may be much higher during a shorter term, as our study found that at $2-3$ years, it diminishes with time. These hypotheses need to be further tested.

The mechanism by which depression with anxiety leads to graft loss post-transplant is not clear. One pathway to mortality could be through non-adherence to the medical regimen and immunosuppressant medication, and we speculate that patients with more distress, such as depression with anxiety, may be at a higher risk for non-adherence $;{ }^{17}$ however, we did not evaluate non-adherence in this review, and this needs to be tested. Another one could include inflammation caused by depression, leading to cardiovascular disease. Depression has been linked to specific pathophysiologic factors including an imbalance between the sympathetic and the parasympathetic systems, sympathoadrenal activation, hypothalamicpituitary-adrenal axis activation, immune system dysregulation with the release of pro-inflammatory cytokines and chemokines, platelet activation, and hypercoagulability. ${ }^{18}$ These hypotheses need to be tested.

A surprising finding of our study was that pre-transplant hypertension was associated with decreased risk of mortality and re-transplant at 2,3, and 5 years post-heart transplant $(\mathrm{HR}=0.2, p \leq 0.03)$. The mechanism for this association is unclear. In renal transplant recipients, one report found that pre-transplant hypertension contributed to chronic progressive graft dysfunction. ${ }^{19}$ One possible pre-transplant explanation is that patients with a pre-transplant history of hypertension tolerate higher doses of angiotensin-converting enzyme inhibitors and $\beta$-blockers and that this confers some "carryover" benefit to the post-transplant period, but there are no current data to support that dosage of these cardiotonic medications influences post-transplant outcomes. Another possible explanation is that patients with preexisting hypertension diagnosis were treated more aggressively for post-transplant hypertension, which is incredibly common post-heart transplant $(71.2 \%$ at 1 year and $91.1 \%$ within 5 years). ${ }^{20}$ Further investigation is needed to evaluate the decreased risk of mortality associated with pre-transplant hypertension as noted by our results.

In the United States, according to the Organ Procurement and Transplantation Network (OPTN)/Scientific Registry of Transplant Recipients (SRTR) 2012 Annual Data Report, graft survival for heart transplants performed from 2005 to 2007 was $88 \%$ at 1 year, $81 \%$ at 3 years, and $75 \%$ at 5 years. According to the ISHLT registries, unadjusted 1-year survival of the most recent cohort of patients transplanted in 2006 to June 2012 was $84 \%$, and survival after re-transplantation was $70 \%$ at 1 year. $^{2}$ In our cohort, overall survival at 1 -year post-heart transplant was $93.1 \%$, therefore higher than the national averages.

We are pleased that our cohort's survival has been higher than the American averages, and we speculate that KPNC's innovative health care delivery system, which includes integrated providers within an EMR system, facilitated our success, however, that needs to be further tested and studied.

Limitations of our study include a small sample size. We were not powered to detect effects individually among depression-only and anxiety-only groups. Small sample is a limitation of virtually all organ transplant studies; the median sample size for studies of depression and anxiety on post-transplant mortality was $147 .{ }^{14}$ Our results may not be generalizable to heart transplant settings that do not work with an integrated care system or settings with different patient demographics. The strengths of the study include an integrated health care delivery system with complete data capture through the EMR and uniform access to care among our patients.

\section{Conclusion}

Our findings suggest an association between preoperative depression with anxiety, with mortality 2 and 3 years after heart transplant, in an integrated health care setting with a diverse patient population. We do not know about the direction of this association and recommend further study to 
uncover it. Given the small sample size and limited number of deaths, the possibility of spurious findings is heightened, and therefore, we recommend that these results be interpreted cautiously. Nevertheless, our results reflect our single cohort's experience, and they add to the existing literature stressing the importance of assessing depression and anxiety before heart transplant, as high distress pre-transplant may be associated with mortality post-transplant.

Future studies should focus on prospective randomized designs, with larger sample sizes, to measure the effect of depression and anxiety pre-heart transplant on morbidity and mortality post-heart transplant and whether behavioral health treatment and psychotropic medication pre-transplant have an impact on survival post-transplant.

\section{Disclosure}

The authors report no conflicts of interest in this work.

\section{References}

1. UNOS [homepage on the Internet]. Organ Procurement and Transplantation Network (OPTN)'s heart 2012 report, within the United Network for Organ Sharing (UNOS). Available from: http://www.unos.org. Accessed February 17, 2017.

2. Lund LH, Edwards LB, Kucheryavaya AY, et al. The registry of the International Society for Heart and Lung Transplantation: thirty-first official adult heart transplant report - 2014; focus theme: retransplantation. J Heart Lung Transplant. 2014;33(10):996-1008.

3. Rugulies R. Depression as a predictor for coronary heart disease: a review and meta-analysis. Am J Prev Med. 2002;23(1):51-61.

4. Olbrisch ME, Benedict SM, Ashe K. Psychological assessment and care of organ transplant patients. J Consult Clin Psychol. 2002;70(3):771-777.

5. Cleland J. Depression Linked to Death in Many Heart Failure Patients. London: Imperial College; 2015.

6. Boyle SH, Samad Z, Becker RC, et al. Depressive symptoms and mental stress-induced myocardial ischemia in patients with coronary heart disease. Psychosom Med. 2013;75(9):822-831.
7. Versteeg H, Hoogwegt M, Hansen T, Pedersen S, Zwisler AD, Thygesen LC. Depression, not anxiety, is independently associated with 5-year hospitalizations and mortality in patients with ischemic heart disease. J Psychosom Res. 2013;75(6):518-525.

8. Faris R, Purcell H, Henein MY, Coats AJ. Clinical depression is common and significantly associated with reduced survival in patients with non-ischemic heart failure. Eur J Heart Fail. 2002;4(4):541-551.

9. Corbett C, Armstrong MJ, Parker R, Webb K, Neuberger JM. Mental health disorders and solid-organ transplant recipients. Transplantation. 2013;96(7):593-600.

10. Engle D. Psychosocial aspects of the organ transplant experience: what has been established, and what we need for the future. J Clin Psychol. 2001;57(4):521-549.

11. Zipfel S, Schneider A, Wild B, et al. Effect of depressive symptoms on survival after heart transplantation. Psychosom Med. 2002; 64(5):740-747.

12. Favaro A, Gerosa G, Caforio AL, et al. Posttraumatic stress disorder and depression in heart transplantation recipients: the relationship with outcome and adherence to medical treatment. Gen Hosp Psychiatry. 2011;33(1):1-7.

13. Owen JW, Bonds CL, Wellisch DK. Psychiatric evaluations of heart transplant candidates: predicting post-transplant hospitalizations, rejection episodes, and survival. Psychosomatics. 2006;47(3):213-222.

14. Dew AM, Rosenberger EM, Myakovsky L, et al. Depression and anxiety as risk factors for morbidity and mortality after organ transplantation: a systematic review and meta-analysis. Transplantation. 2015;100(5):988-1003.

15. Beck AT, Steer RA, Brown GK. Beck Depression Inventory: BDI-II. 2nd ed. San Antonio, TX: Harcourt Brace \& Company; 1996.

16. Sears FS, Rodrigue JR, Sirois BC, Urizar GG, Perri MG. Extending psychometric norms for pre-cardiac transplantation evaluations: The Florida Cohort, 1990-1996. J Clin Psychol Med Sett. 1999;6(3):303-316.

17. Cukor D, Newville H, Jindal R. Depression and immunosuppressive medication adherence in kidney transplant patients. Gen Hosp Psychiatry. 2008;30(4):386-387.

18. Halaris A. Inflammation, heart disease, and depression. Curr Psychiatry Rep. 2013;15(10):400.

19. Frei U, Schindler R, Wieters D, Grouven U, Brunkhorst R, Koch KM. Pre-transplant hypertension: a major risk factor for chronic progressive renal allograft dysfunction? Nephrol Dial Transplant. 1995; 10(7):1206-1211.

20. Lund LH, Edwards LB, Kucheryavaya AY, et al. The Registry of the International Society for Heart and Lung Transplantation: thirty-second official adult heart transplantation report-2015. Focus theme: early graft failure. J Heart Lung Transplant. 2015;34(10):1244-1254.
Transplant Research and Risk Management

\section{Publish your work in this journal}

Transplant Research and Risk Management is an international, peerreviewed open access journal focusing on all aspects of transplantation and risk management to achieve optimal outcomes in the recipient improving survival and quality of life. The manuscript management system is completely online and includes a very quick and fair peer-review system, which is all easy to use. Visit http://www.do
to read real quotes from published authors.

\section{Dovepress}

Submit your manuscript here: https://www.dovepress.com/transplant-research-and-risk-management-journal 